\title{
Effects of carprofen or meloxicam on selected haemostatic variables in miniature pigs after orthopaedic surgery
}

\author{
Petr Raušer ${ }^{1}$, Leona Lexmaulová ${ }^{1}$, Robert Srnec $^{1}$, Lucie Urbanová ${ }^{1}$, Pavel Proks ${ }^{2}$, \\ Alois Nečas ${ }^{1}$
}

\begin{abstract}
${ }^{1}$ Department of Surgery and Orthopaedics, ${ }^{2}$ Department of Diagnostic Imaging, Small Animal Clinic, Faculty of Veterinary Medicine, University of Veterinary and Pharmaceutical Sciences Brno, Czech Republic
\end{abstract}

Received October 12, 2010

Accepted October 11, 2011

\begin{abstract}
The aim of the study was to detect and compare the haemostatic variables and bleeding after 7-days administration of carprofen or meloxicam in clinically healthy miniature pigs. Twenty-one clinically healthy Göttingen miniature pigs were divided into 3 groups. Selected haemostatic variables such as platelet count, prothrombin time, activated partial thromboplastin time, thrombin time, fibrinogen, serum biochemical variables such as total protein, bilirubin, urea, creatinine, alkaline phosphatase, alanine aminotransferase and gamma-glutamyltransferase and haemoglobin, haematocrit, red blood cells, white blood cells and buccal mucosal bleeding time were assessed before and 7 days after daily intramuscular administration of saline $\left(1.5 \mathrm{ml}\right.$ per animal, control group), carprofen $\left(2 \mathrm{mg} \cdot \mathrm{kg}^{-1}\right)$ or meloxicam $\left(0.1 \mathrm{mg} \cdot \mathrm{kg}^{-1}\right)$. In pigs receiving carprofen or meloxicam, the thrombin time was significantly increased $(p<0.01)$ compared to the control group. In animals receiving carprofen, serum urea and creatinine were significantly decreased, compared to the control $(p<0.01)$ or meloxicam $(p<0.05)$ groups. In pigs receiving meloxicam, serum urea was significantly decreased $(p<0.05)$ compared to the control group. Significant differences were not detected in other haemostatic, biochemical variables or bleeding time compared to other groups or to the pretreatment values. Intramuscular administration of carprofen or meloxicam in healthy miniature pigs for 7 days causes sporadic, but not clinically important changes of selected haemostatic variables. Therefore, we can recommend them for perioperative use, e.g. for their analgesic effects, in orthopaedic or other surgical procedures without increased bleeding.
\end{abstract}

Swine, non-steroidal anti-inflammatory drugs, thrombin time

Surgeries often lead to tissue damage associated with inflammation. High-quality analgesia therefore forms an integral part of any surgery (L e m ke 2004). In order to ensure also an anti-inflammatory effect of analgesics, nonsteroidal antiinflammatory drugs (NSAIDs) are a suitable choice. Nonsteroidal antiinflammatory drugs often have to be administered for a long period of time in dependence on the extent of tissue damage and duration of the pain process.

In dogs and cats, carprofen and meloxicam are frequently used for these purposes. There are many reports available on both the desired and adverse effects of these preparations (Lascelles 2000; Matthews 2000). In most cases, NSAIDs represent drugs of choice (Nolan and Reid 1993; Lascelles et al. 1994) due to the low rate of adverse effects (Forsyth et al. 1998; Reimer et al. 1999). However, data on the use of meloxicam or carprofen in pigs are sporadic (Swindle 2007); no data at all are available on their longterm administration.

Nonsteroidal antiinflammatory drugs inhibit enzyme cyclooxygenase (COX) that catalyzes reactions leading to production of prostaglandins and thromboxanes (Papich 1997). Production of thromboxanes by A2 thrombocytes is important for primary haemostasis (Relford 1992; Schafer 1995). Administration of NSAIDs can therefore be associated with haemostatic dysfunction. Nonsteroidal antiinflammatory drugs that 
irreversibly inhibit activity of COX (Donnelly and Hawkey 1997) can increase the risk of haemorrhage (Schafer 1995; Nolte et al. 1997). Acetylsalicylic acid induces dysfunction of thrombocytes that lasts throughout their lifespan (Vane 1987), however, carprofen inhibits thrombocyte function only temporarily during its administration and some time after its discontinuation (Grisneaux et al. 1999).

Hickford et al. (2000) did not observe any clinically significant effects of carprofen on haemostasis in dogs. Buccal mucosal bleeding time and clotting ability (McKellar et al. 1990) were not affected by administration of carprofen. McKellar et al. (1990, 1994) monitored thromboxane B2 as an indicator of COX activity of platelets and found that in vitro administration of carprofen did not impair the platelets' production of thromboxane B2.

The aim of this study was to compare the effect of administration of carprofen and meloxicam in miniature pigs on selected haemostatic and biochemical blood variables that could indicate impaired coagulation. Unlike previous studies, this study investigated the effect of NSAIDs administered for a longer period of time (1 week), which should suggest possibilities of prolonged NSAID therapy in miniature pigs. Prolonged therapy is desirable mainly for postoperative care, e.g. in orthopaedic or other surgical procedures.

\section{Materials and Methods}

Twenty-one clinically healthy Göttingen miniature pigs, females weighing $35.3 \pm 7.10 \mathrm{~kg}$ (mean $\pm \mathrm{SD}$ ) and aged $2.2 \pm 0.74$ years were used for the study. All animals had identical housing, feeding and unlimited water intake. During the last 3 months before experiment, they were without any medication or surgery. All pigs served as control groups for an experimental study of the healing of fracture defects using transplantation of different scaffolds in combination with mesenchymal stem cells (NPV II Research Project 2B06130).

Animals were divided into three groups: CAR, MEL and SAL (control group), each containing 7 pigs. Carprofen $2 \mathrm{mg} \cdot \mathrm{kg}^{-1}$ (Rimadyl; Vericore Ltd., U.K.), meloxicam $0.1 \mathrm{mg} \cdot \mathrm{kg}^{-1}$ (Metacam; Labiana Pharm., Spain) and saline $0.9 \% 1.5 \mathrm{ml}$ was administered per animal in CAR, MEL and SAL groups, respectively. Each pig received drugs intramuscularly (i.m.) once a day (at 13:00 h) for 7 days at the above dose.

Blood samples were collected from all pigs of all 3 groups at the beginning of the experiment and 7 days after medication. A total of $4 \mathrm{ml}$ of blood was collected by venipuncture from the jugular vein into a polypropylene syringe. An $0.9 \mathrm{ml}$ of blood was transferred into a polypropylene tube containing $0.1 \mathrm{ml}$ of sodium citrate for a coagulation panel: prothrombin time (PT), activated partial thromboplastin time (APTT), thrombin time (TT) and fibrinogen. A $1.0 \mathrm{ml}$ of blood was transferred into tube with EDTA for measurement of haemoglobin $(\mathrm{Hb})$, haematocrit (Hct), red blood cells (RBC) white blood cells (WBC) and platelet count (Thromb). Two ml of blood were transferred into a polypropylene tube for measurement of serum biochemical variables: total protein (TP), bilirubin (BIL), urea (U), creatinine (CR), alkaline phosphatase (ALP), alanine aminotransferase (ALT) and gamma-glutamyltransferase (GGT).

The $\mathrm{Hb}, \mathrm{Hct}, \mathrm{RBC}, \mathrm{WBC}$ and Thromb were determined using an automated haematologic analyzer (Celltac alpha MEK 6318, Nihon Kohden, Japan), coagulation panel PT, APTT, TT and fibrinogen concentration by the use of coagulometer (Coatron M2 - Teco, Germany) and biochemical variables by the automated biochemic analyzer (DPC Konelab 20i, Thermo Scientific, Finland).

For bleeding test, all pigs were sedated before and 7 days after carprofen, meloxicam or saline administration by i.m. administration of a mixture of tiletamine-zolazepam $2 \mathrm{mg} \cdot \mathrm{kg}^{-1}$ (Zoletil 100, Virbac, France), xylazine $0.4 \mathrm{mg} \cdot \mathrm{kg}^{-1}$ (Rometar, Bioveta, Czech Republic) and ketamine $2 \mathrm{mg} \cdot \mathrm{kg}^{-1}$ (Narketan, Vetiquinol, France). Bleeding test was performed exactly $30 \mathrm{~min}$ after sedation. Each pig was positioned in lateral recumbency. The upper lip was tied so that the buccal mucosal surface was exposed. With a scalpel blade (No 11) two simultaneous parallel incisions of standardized length and depth in an area of the mucosa free of obvious blood vessels were performed. At intervals of 5 seconds, No. 1 Whatman filter paper was used to blot away the blood, taking care not to touch the wound edges. Using a stopwatch, the time from creating the incisions until bleeding ceased was recorded for each incision, and the mean value (seconds) was recorded as the final BT.

Variables of each group of animals 7 days after medication were compared with variables before the experiment. Thereafter, variables evaluated 7 days after medication were compared with each group (CAR vs. MEL, CAR vs. SAL, MEL vs. SAL). All variables were compared by means Kruskal-Wallis and a Wilcoxon test. In all analyses, a value of $p<0.05$ was considered significant.

\section{Results}

In pigs receiving carprofen or meloxicam, the TT was significantly increased $(p<0.01)$ compared to other groups. In animals receiving carprofen, serum $\mathrm{U}$ and $\mathrm{CR}$ were significantly decreased, compared to the control $(p<0.01)$ or meloxicam $(p<0.05)$ groups. 
In pigs receiving meloxicam, serum $\mathrm{U}$ was significantly decreased $(p<0.05)$ compared to the control group (Table 1).

Table 1. Comparison of haemostatic and selected serum blood variables of pigs before and 7 days after administration of saline (SAL), carprofen (CAR) or meloxicam (MEL)

\begin{tabular}{|c|c|c|c|c|c|}
\hline Variable & Group & Range & Median & Analysis & $P$ value \\
\hline \multirow[t]{6}{*}{ TT (s) } & SAL-0 & $18.7-29.8$ & 26.8 & CAR-7 vs. SAL-7 & $0.0091 * *$ \\
\hline & CAR-0 & $18.9-27.2$ & 25.8 & MEL-7 vs. SAL-7 & $0.0054 * *$ \\
\hline & MEL-0 & $19.0-29.2$ & 26.1 & CAR-7 vs. CAR-0 & $0.0087 * *$ \\
\hline & SAL-7 & $18.8-29.7$ & 26.9 & MEL-7 vs. MEL-0 & $0.0054 * *$ \\
\hline & CAR-7 & $27.3-68.3$ & 65.3 & & \\
\hline & MEL-7 & $40.0-108.7$ & 83.3 & & \\
\hline \multirow[t]{6}{*}{ Serum U (mmol/l) } & SAL-0 & $2.7-5.8$ & 4.9 & CAR-7 vs. SAL-7 & $0.0046 * *$ \\
\hline & CAR-0 & $2.9-6.2$ & 4.8 & MEL-7 vs. SAL-7 & $0.0328 *$ \\
\hline & MEL-0 & $2.9-6.0$ & 4.8 & CAR-7 vs. MEL-7 & $0.0291 *$ \\
\hline & SAL-7 & $3.5-7.0$ & 5.8 & & \\
\hline & CAR-7 & $2.4-4.5$ & 3.4 & & \\
\hline & MEL-7 & $4.2-6.8$ & 3.9 & & \\
\hline \multirow[t]{6}{*}{ Serum CR $(\mu \mathrm{mol} / \mathrm{l})$} & SAL-0 & $76.5-141.3$ & 106.7 & CAR-7 vs. SAL-7 & $0.0032 * *$ \\
\hline & CAR-0 & $80.1-128.8$ & 111.2 & CAR-7 vs. MEL-7 & $0.0221 *$ \\
\hline & MEL-0 & $65.9-132.3$ & 114.9 & & \\
\hline & SAL-7 & $113.2-141.3$ & 124.2 & & \\
\hline & CAR-7 & $61.0-90.8$ & 74.1 & & \\
\hline & MEL-7 & $89.6-154.6$ & 99.7 & & \\
\hline
\end{tabular}

Significant difference $* p<0.05$ or $* * p<0.01$ between values for the times and groups compared. SAL-0, CAR-0, MEL-0 - pigs before medication, SAL-7, CAR-7, MEL-7 - pigs 7 days after administration of saline, carprofen or meloxicam, TT - thrombin time, $\mathrm{U}$ - urea, $\mathrm{CR}$ - creatinine

Significant differences were not detected in other coagulation panel parameters (PT, APTT, fibrinogen), bleeding time, platelet count, Hb, Hct, RBC, WBC or serum biochemical variables (TP, BIL, ALP, ALT, GGT).

\section{Discussion}

Impairment of coagulation in miniature pigs after administration of carprofen or meloxicam can be induced by various factors, wrong technique of taking and processing samples being one of them (Vaden et al.2009). Blood was taken lege artis using commercial test tubes filled with precisely defined volume of citrate. In all cases, tubes were filled with sufficient amount of blood to avoid subsequent misinterpretation of laboratory analysis. All samples were processed immediately as per standard laboratory procedures. Errors in preanalytic and analytic phases causing the observed abnormalities thus can be ruled out.

Abnormalities may have occurred also due to administration of carprofen or meloxicam. Many publications describe the possible negative effect of NSAIDs on platelet functions (Vane 1987; Grisneaux et al. 1999; Blois et al. 2010). Haemostasis and formation of blood clot after vessel damage result from interaction between vessel wall, platelets and clotting factors. This is a rather complicated process controlled by several systems including metabolization of arachidonic acid that can be affected by administration of NSAIDs (Papich 1997; Triplett 2000). Platelets participate not only in primary haemostasis, but also actively influence secondary haemostasis which is based on interaction between clotting factors (Triplett 2000). 
Rogers and Levin (1990) desribe buccal mucosal bleeding time as a relatively insensitive screening test. However, McKellar et al. (1990) did not observe any change of this parameter after administration of carprofen in dogs. Our results correspond with their study, as we also did not find any change of this parameter in pigs after administration of carprofen or meloxicam. Unlike McKellar, we were not able to monitor values of thromboxane B2 as an indicator of COX activity of platelets (McKellar et al. 1990, 1994). We also did not detect significant APTT and PT deviations in control animals after administration of carprofen or meloxicam. Similar conclusions were drawn by Hickford et al. (2000) who evaluated the effect of carprofen on haemostasis in dogs.

Thrombin time was the only haemostatic parameter that was significantly increased in miniature pigs after carprofen or meloxicam administration. Prolonged TT in miniature pigs medicated with carprofen or meloxicam could be determined by several factors: hypofibrinogenaemia, dysfibrinogenaemia, or administration of substances inhibiting thrombin (Vaden et al. 2009). As no significant differences in fibrinogen concentrations between carprofen/meloxicam-medicated animals and control animals were found, hypofibrinogenaemia as the cause of prolonged TT can be ruled out. Inhibition of thrombin is another suggested cause of prolonged TT. Thrombin can be inhibited e.g. by heparin, fibrin degradation products, or indirectly by inhibited platelet function (Triplett 2000). None of the animals enrolled in our study was medicated with heparin, so heparin can be ruled out as the cause of prolonged TT in miniature pigs receiving carprofen or meloxicam. Prolonged TT in animals from our study could be caused by increased concentration of fibrin degradation products that are metabolized and degraded in liver, or by altered activity of thrombin resulting from carprofen or meloxicam action. Fibrin degradation products are captured and eliminated in the liver (Mueller et al. 2002). Administration of NSAIDs can have a negative impact on liver function, which leads to decreased ability to capture and degrade fibrin degradation products (FDP). Although FDP concentrations were not evaluated in our study, biochemical examination of blood (TP, BIL, U, CR, ALP, ALT, GGT) indicates that severe impairment of liver function can be excluded (Mueller et al. 2002; Vaden et al. 2009).

Inhibition of thrombin through the effect on platelets seems to be the most likely cause of prolonged TT in miniature pigs medicated with carprofen or meloxicam. During clotting process, activation of platelets occurs with expression of negatively charged phospholipids, which leads to accumulation of clotting factors on membranes of activated platelets. Afterwards, thrombokinase is released, which in synergy with certain clotting factors (factors $\mathrm{V}, \mathrm{X}$ ) leads to transformation of inactive prothrombin to active thrombin. Production of thrombin is further escalated by activation of factors V, VIII and XI, leading to transformation of soluble fibrinogen to insoluble fibrin (Butenas and Mann 2001). As interaction between individual components of the coagulation system is regulated, among others, by metabolization of arachidonic acid, it can be assumed that administration of carprofen or meloxicam in miniature pigs interfered with this regulation, which influenced thrombin activation and thus also prolonged TT. Exact verification of this hypothesis will be the subject of future studies.

Lower concentrations of urea and creatinine in blood of miniature pigs receiving carprofen or meloxicam could be associated with analgesia. Assumably, carprofen and meloxicam have a positive effect in that they suppress inflammatory response and thus also reduce pain after surgeries such as transplantations or bone or joint interventions. Compared to animals without analgesia, carprofen/meloxicam-medicated animals showed a better intake of feed and water, which prevented limitation of liver functions. This fact corresponds with results of studies on the negative effect of pain on animals (Lascelles 2000).

Intramuscular administration of carprofen or meloxicam in healthy miniature pigs for 7 days causes sporadic, but not clinically important changes of selected haemostatic 
variables. These drugs only increase thrombin time and decrease serum concentrations of urea and creatinine. When carprofen or meloxicam are used in pigs before surgery, e.g. for their analgesic effects, they should not affect the patient's haemostatic properties during the surgery.

\section{Acknowledgements}

This work was supported by the Ministry of Education, Youth and Sports of the Czech Republic (NPV II Research Project 2B06130).

\section{References}

Blois SL, Allen DG, Wood RD, Conlon PD 2010: Effects of aspirin, carprofen, deracoxib, and meloxicam on platelet function and systemic prostaglandin concentrations in healthy dogs. Am J Vet Res 71: 349-358

Butenas S, Mann KG 2001: Blood coagulation. Biochemistry (Moscow) 67: 3-12

Donnelly MT, Hawkey CJ 1997: COX-II inhibitors - a new generation of safer NSAIDs? Aliment Pharmacol Ther 11: 227-236

Forsyth SF, Guilford WG, Haslett SJ, Godfrey J 1998: Endoscopy of the gastroduodenal mucosa after carprofen, meloxicam and ketoprofen administration in dogs. J Small Anim Pract 39: 421-424

Grisneaux E, Pibarot P, Dupuis J, Blais D 1999: Comparison of ketoprofen and carprofen administered prior to orthopedic surgery for control of postoperative pain in dogs. J Am Vet Med Assoc 215: 1105-1110

Hickford FH, Barr SC, Erb HN 2000: Effect of carprofen on hemostatic variables in dogs. Am J Vet Rec 62: $1642-1646$

Lascelles BDX, Butterworth SJ, Waterman AE 1994: Postoperative analgesic and sedative effects of carprofen and pethidine in dogs. Vet Rec 134: 187-191

Lascelles DX (2000): Clinical pharmacology of analgesic agents. In: Hellebrekers LJ (eds.), Animal pain. Van der Wees, Utrecht 85-116

Lemke KA 2004: Understanding the pathophysiology of perioperative pain. Can Vet J 45: 475-480

Matthews KA 2000: Management of pain. Vet Clin North Am Small Anim Pract 30: 703-970

McCrory CR, Lindahl SG 2002: Cyclooxygenase inhibition for postoperative analgesia. Anesth Analg 95: 169-176

McKellar QA, Delatour P, Lees P 1994: Stereospecific pharmacodynamics and pharmacokinetics of carprofen. J Pharm Sci 17: 447-454

McKellar QA, Pearson T, Bogan JA 1990: Pharmacokinetics, tolerance and serum thromboxane inhibition of carprofen in the dog. J Small Anim Pract 31: 443-448

Mueller MM, Bomke B, Seifried E 2002: Fresh frozen plasma in patients with disseminated intravascular coagulation or in patients with liver diseases. Thromb Res 107: 9-17

Nolan AM, Reid J 1993: Comparison of the postoperative analgesic and sedative effects of carprofen and papaveretum in the dog. Vet Rec 133: $240-242$

Nolte I, Niemann C, Bowry SK, Failing K, Müller-Berghaus K 1997: A method for measuring capillary bleeding time in non-anaesthetized dogs: prolongation of the bleeding time by acetylsalicylic acid. Zentralbl Veterinärmed 44: 625-628

Papich MG 1997: Principles of analgesic drug therapy. Semin Vet Med Surg 12: 80-93

Relford R 1992: Diagnosis of platelet disorders. Semin Vet Med Surg 7: 323-329

Rodgers RP, Levin J 1990: A critical reappraisal of the bleeding time. Semin Thromb Hemost 16: 1-20

Schafer AI 1995: Effects of nonsteroidal antiinflammatory drugs on platelet function and systemic hemostasis. J Clin Pharmacol 35: 209-219

Swidle MM 2007: Swine in the laboratory. Surgery, anesthesia, imaging, and experimental techniques. $2^{\text {nd }}$ ed. CRC Press, Charleston, pp. 35-79

Triplett DA 2000: Coagulation and bleeding disorders: Review and Update. Clin Chem 46: 1260-1269

Vaden SL, Knoll JS, Smith FWK, Tilley LP 2009: Blackwel's five-minute veterinary consult: Laboratory tests and diagnostic procedures. Canine \& Feline. Willey-Blackwell, Ames, 763 p.

Vane 1987: The evolution of non-steroidal anti-inflammatory drugs and their mechanisms of action. Drugs 33: $18-27$ 